\title{
An Intimal Relationship Between Atherosclerosis and Colorectal Cancer Screening
}

\author{
Fredy Nehme ${ }^{1}$
}

Published online: 6 January 2020

(c) Springer Science+Business Media, LLC, part of Springer Nature 2020

CRC is one of the most preventable and treatable cancers affecting adults if detected early. Despite continuous efforts directed toward reducing colorectal cancer (CRC) incidence over the past several years, CRC remains the second leading cause of cancer-related mortality in the USA [1]. Although current guidelines recommend CRC screening for adults based on age and family history, several other genetic, environmental, and lifestyle factors have been implicated in CRC development. In fact, a worrying rise in CRC is noted in patients younger than 50 years, prompting the quest to discover novel tools that could be used to select patients who would benefit the most or earlier from screening [2]. Risk prediction models have been developed to stratify populations at low and high risk of CRC development, moving a step forward toward the implementation of precision medicine [3]. Stratification increases both screening adherence and efficiency, since higher-risk individuals are more likely to undergo surveillance when made aware of their increased risk [1] while optimizing utilization of available resources. Although risk factors for CRC have been extensively studied, translating these factors into clinically meaningful tools has been challenging.

In this issue of Digestive Diseases and Sciences, Kim and colleagues evaluated the association between a surrogate marker for atherosclerosis (assessed using carotid ultrasonography) and colorectal adenomas [4]. In this study, asymptomatic adults aged $\geq 40$ years in Korea were offered health screening packages that included carotid ultrasound and colonoscopy. Those who chose these two modalities as part of their routine check-up were included in the study in which the data were collected prospectively. Patients who

Guarantor: Fredy Nehme, MD

Fredy Nehme

nehme.fredy@gmail.com

1 Department of Gastroenterology and Hepatology, University of Missouri Kansas City, 5000 Holmes St, Kansas City, MO 64110, USA had undergone colonoscopy within the past 5 years and had a history of CRC, inflammatory bowel disease, or known cardiovascular disease were excluded. A total of 4781 participants were included in the final analysis. Carotid intima media thickness (CIMT) $\geq 1 \mathrm{~mm}$ and the presence of carotid plaque were used as surrogate markers of subclinical atherosclerosis. High-risk adenomas were defined as any adenoma $>1 \mathrm{~cm}$, the presence of predominant villous features, the presence of high-grade dysplasia, or $\geq 3$ adenomas.

Individuals with abnormal CIMT or the presence of plaque had a significantly higher prevalence of average- and high-risk adenomas than those with normal CIMT or the absence of plaque. Multivariable analysis confirmed both surrogate markers as independent risk factors for conventional and high-risk adenomas.

CRC and atherosclerosis share several established common risk factors including smoking, type 2 diabetes, and obesity [5]. Therefore, the association between colorectal adenomas and abnormal carotid ultrasound findings is not surprising. Though it has been previously reported, this is the largest study to examine this association adding to the growing body of evidence on this topic. In fact, atherosclerosis and colorectal adenomas may share a common pathogenesis of chronic inflammation. While atherosclerosis is associated with chronic inflammation [6], there is also a growing literature implicating inflammation in the development of colorectal adenomas. Data supporting this concept include the protective effect of nonsteroidal antiinflammatory drugs on CRC [7], the association between elevated C-reactive protein and incident CRC [8], and shared molecular mechanisms. Moreover, medications traditionally used to prevent the development of atherosclerotic disease such as statins and aspirin appear to help prevent CRC [9].

Using a noninvasive, easily accessible, and relatively inexpensive test such as carotid ultrasonography, this study translates this association into clinical practice. In fact, individuals aged 40-49 with abnormal surrogate markers for atherosclerosis had comparable rates of average- and 
high-risk adenomas as did those aged 50-59 with normal markers irrespective of family history of CRC. This suggests that carotid atherosclerosis may be used as marker to risk stratify patients at higher risk of CRC who could benefit from earlier screening. Yet, the clinical applicability of these findings remains in question. The U.S. Preventive Services Task Force (USPSTF) does not recommend the routine use of duplex ultrasonography to detect asymptomatic carotid stenosis given the limited overall benefit in detecting carotid stenosis in these individuals [10]. While this test appears to be commonly offered in Korea for asymptomatic individuals, routine use is currently limited in Western countries. Although a simple, noninvasive test such as carotid ultrasound appears to be an attractive tool for risk stratification in CRC screening, many questions remain to be answered including cost-effectiveness, and possible harms of interventions for asymptomatic patients diagnosed with carotid artery stenosis before its routine use in clinical practice.

As the authors acknowledge, there are limitations to this study. This is a single-center retrospective analysis of prospectively collected data of patients who volunteered for routine health check-ups, a group that may not represent the general population. Furthermore, the current study along with others examining this association was all conducted in Korea, further limiting the generalizability of these findings. Also, data detailing the results of previous colonoscopies performed $>5$ years prior to study onset were not available. Despite some limitations, this study was strengthened by the large number of study participants and the systematic and subgroup analysis performed.

To note, the prevalence of CRC did not differ between participants with normal and abnormal surrogate markers for carotid atherosclerosis. Still, since only 19 patients were found to have CRC without previously known atherosclerotic disease, conclusions cannot be drawn given this small number.

Finally, using age and family history as the only factors to determine the starting age for CRC screening may not be sufficient. Risk prediction models including genetic variants, lifestyle, and environmental factors have been studied to accurately stratify patients based on their risk [3]. Carotid atherosclerosis may contribute to future prediction models aimed at better prioritizing those at high risk of targeted CRC prevention. While Kim and colleagues should be commended for their work in highlighting the association between carotid atherosclerosis and colorectal adenomas, the generalizability of these findings in non-Asian populations and the cost-effectiveness of routine carotid ultrasound remain in question, requiring further studies to address these issues. Given the suspected common pathogenesis of these two entities, simple inflammatory biomarkers such as C-reactive protein may be important components of scoring systems use to stratify patients for CRC screening.

Funding None.

\section{Compliance with Ethical Standards}

Conflict of interest This statement is to certify that the author has no conflict of interest related to the current manuscript.

\section{References}

1. Lieberman D, Ladabaum U, Cruz-Correa M, et al. Screening for colorectal cancer and evolving issues for physicians and patients: a review. JAMA. 2016;316(20):2135-2145.

2. Bailey CE, Hu C-Y, You YN, et al. Increasing disparities in the age-related incidences of colon and rectal cancers in the United States, 1975-2010. JAMA Surg. 2015;150(1):17-22.

3. Jeon J, Du M, Schoen RE, et al. Determining risk of colorectal cancer and starting age of screening based on lifestyle, environmental, and genetic factors. Gastroenterology. 2018;154(8):2152. e19-2164.e19.

4. Kim J, Lee JY, Ham NS, et al. Association between carotid ultrasonography findings and colorectal adenoma in asymptomatic adults. Dig Dis Sci. (Epub ahead of print). https://doi.org/10.1007/ s10620-019-05899-7.

5. Dekker E, Tanis PJ, Vleugels JLA, Kasi PM, Wallace MB. Colorectal cancer. Lancet Lond Engl. 2019;394(10207):1467-1480.

6. Libby P. Inflammation in atherosclerosis. Nature. 2002;420(6917):868-874.

7. Arber N, Levin B. Chemoprevention of colorectal neoplasia: the potential for personalized medicine. Gastroenterology. 2008;134(4):1224-1237.

8. Tsilidis KK, Branchini C, Guallar E, Helzlsouer KJ, Erlinger TP, Platz EA. C-reactive protein and colorectal cancer risk: a systematic review of prospective studies. Int $J$ Cancer. 2008;123(5):1133-1140.

9. Dobrzycka M, Spychalski P, Łachiński AJ, Kobiela P, Jędrusik P, Kobiela J. Statins and colorectal cancer-a systematic review. Exp Clin Endocrinol Diabetes Off J Ger Soc Endocrinol Ger Diabetes Assoc. 2018; https://doi.org/10.1055/a-0668-5692.

10. Jonas DE, Feltner C, Amick HR, et al. Screening for asymptomatic carotid artery stenosis: a systematic review and meta-analysis for the U.S. Preventive Services Task Force. Ann Intern Med. 2014;161(5):336-346.

Publisher's Note Springer Nature remains neutral with regard to jurisdictional claims in published maps and institutional affiliations. 